\title{
Retención de vitamina C en el procesamiento de la pulpa de copoazú (Theobroma grandiflorum) enlatada
}

\section{(Retention of vitamin $\mathrm{C}$ in the processing of canned cupuassu pulp (Theobroma grandiflorum))}

\author{
Robert Tecse-Tecsi', Virne Mego-Mego², Miguel Chávez-Pinchi', Lastenia Cutipa-CháveZt, Luis \\ Vargas-Vásquez ${ }^{5}$
}

\begin{abstract}
Resumen
La pulpa de copoazú es un fruto de alto contenido en pectinas y ácido ascórbico cosechado en la Amazonía de América Oriental por sus características de aroma, sabor y textura. No obstante, el procesamiento de enlatado para el consumo humano en Madre de Dios (Perú) es cuestionable debido al inadecuado manejo y tratamiento térmico del fruto. El objetivo del estudio fue evaluar la estabilidad de la conservación de la vitamina C en el procesamiento de la pulpa de copoazú enlatada. Para ello, se llevó a cabo un diseño experimental en cuatro etapas: 1) caracterización del fruto, 2) determinación del tratamiento térmico, 3) análisis de la variación de vitamina C y 4) análisis de la pulpa enlatada. Se evaluó el efecto del proceso por triplicado y se aplicó un diseño completamente al azar (DCA) para analizar las diferencias estadísticas mediante el programa Statgraphys Centurion. Entre los resultados se destaca que el pH $(3.05 \pm 0.50)$ y acidez $(1.54 \pm 0.80)$ del enlatado guardan semejanza con la materia prima. Asimismo, se encontró un aumento significativo de sólidos solubles (Brix) respecto al fruto fresco. Se concluye que la retención total de vitamina C de la pulpa de copoazú enlatada fue $49.10 \%$.
\end{abstract}

\section{Palabras clave}

Theobroma grandiflorum, ácido ascórbico, pasteurización, enlatado, tratamiento térmico

\begin{abstract}
The cupuassu pulp is a fruit with a high content of pectins and ascorbic acid harvested in the Amazon of Eastern America for its exquisite characteristics of aroma, flavor and texture; however, the processing of canned food for human consumption in Madre de Dios - Peru is questionable, due to the inadequate handling and heat treatment of the fruit. The objective of the study was to evaluate the stability of the preservation of vitamin $C$ in the processing of canned cupuassu pulp. For this, an experimental design was carried out in four stages: 1) characterization of the fruit, 2) determination of the heat treatment, 3) analysis of the variation of vitamin $C$ and 4) analysis of the canned pulp. The effect of the process was evaluated in triplicate and a Completely Random Design was applied to analyze the statistical differences using the Statgraphys Centurion program. Among the results, it stands out that the $\mathrm{pH}$ (3.05 \pm 0.50$)$ and acidity (1.54 \pm 0.80$)$ of the canning are similar to the raw material. Likewise, a significant increase in soluble solids (Brix) was found with respect to fresh fruit. It is concluded that the total retention of vitamin $C$ of the canned cupuassu pulp was $49.10 \%$.
\end{abstract}

\section{Keywords}

Theobroma grandiflorum; ascorbic acid; pasteurization; canned; heat treatment

\footnotetext{
1 Universidad Nacional Amazónica de Madre de Dios. Puerto Maldonado, Perú. [06210027@unamad.edu.pe, https://orcid. org/0000-0003-3768-1033]

2 Universidad Nacional Amazónica de Madre de Dios. Puerto Maldonado, Perú. [vmego@unamad.edu.pe, https://orcid. org/0000-0001-5800-7430]

3 Universidad Nacional Amazónica de Madre de Dios. Puerto Maldonado, Perú. [mchavez@unamad.edu.pe, https://orcid. org/0000-0001-7431-5871]

$4 \quad$ Universidad Nacional Amazónica de Madre de Dios. Puerto Maldonado, Perú. [lcutipa@unamad.edu.pe, https://orcid. org/0000-0003-3341-1095]

5 Universidad Nacional de San Martín. Tarapoto, Perú. [lmvargas@unsm.edu.pe, https://orcid.org/0000-0003-4418-107X]
} 


\section{Introducción}

Copoazú (Theobroma grandiflorum) es una palabra compuesta que proviene de la lengua tupi, kupu significa "similar al cacao" y uasu "genial" (Gondim et al., 2001). El copoazú es un fruto del mismo género del cacao que se destaca, en la Amazonía de América Oriental, por su potencial económico en el mercado nacional e internacional (Ramos et al., 2020) al ser utilizado como ingrediente de bebidas de zumo de frutas, mermeladas, gelatinas, caramelos y productos lácteos.

El creciente interés económico en este fruto ha generado el desarrollo de nuevas industrias y ha impulsado investigaciones en el sector alimenticio (Ana L. F. Pereira et al., 2018). Esto se debe a sus características relacionadas con el aroma, el sabor, la textura y el alto contenido de pectinas de la pulpa (Maciel et al., 2020). Así lo revelaron Costa et al. (2015), quienes demostraron que los polisacáridos de la pectina del copoazú mejoran la textura de yogures de leche de cabra, por lo que se ha considerado su utilidad en la industria caprina lechera. El estudio de Pereira et al. (2017) es otro ejemplo. Los autores observaron que la pulpa es propicia para el desarrollo de probióticos, ya que presentó ventaja tecnológica como sustrato para la fermentación probiótica, debido a su composición de azúcares naturales y ácidos orgánicos.

La pulpa de copoazú representa el 38 \%-43 \% de la fruta y es rica en ácido ascórbico (vitamina C), con $102 \mathrm{mg} / 100 \mathrm{~g}$. Contiene alta concentración de compuesto fenólicos (3.5$4.9 \mathrm{mg}$ de equivalente de catequina/g de muestra de peso seco), actividad antioxidante (1.7$2.0 \mu \mathrm{M}$ Trolox/g) y potencial de fibra dietética (0.50 \%-2.12 \%) (Pugliese et al., 2013; Salgado et al., 2011). La pulpa que rodea la semilla puede ser extraída de forma manual o mecánica, y es procesada mediante pasteurización, enlatado y/o congelado (Gondim et al., 2001).

Durante este proceso, las vitaminas hidrosolubles de la pulpa de copoazú presentan cambios, sobre todo la vitamina C. Esta es lábil en presencia de oxígeno y rápida en oxidación a temperaturas elevadas (Badui Dergal, 2006; Bouzari et al., 2015). Estos factores repercuten en la calidad organoléptica y son precursores de cambios de la actividad antioxidante que reducen el valor nutricional del fruto (Amit et al., 2017; Cole et al., 2018).

La Amazonía peruana de Madre Dios, ámbito geográfico de este estudio, produce frutales de copoazú a gran escala para el consumo humano y para fines comerciales (Peña Valdeiglesia $\&$ Alegre Orihuela, 2017). Entre los productos elaborados en la región se destaca el enlatado en almíbar de la pulpa; sin embargo, se ha identificado que el proceso de la manufactura no brinda cuidado o importancia al de enlatado, por lo que es cuestionable el tratamiento del fruto para conversar sus valores nutritivos.

La etapa de pasteurización es determinante para elaborar estos alimentos, porque en ella se evalúa el tratamiento térmico óptimo para producir productos inocuos que favorezcan la conservación de vitamina C (Ghoshal, 2018). Por ello, es reconocido que el inadecuado proceso de enlatado de la pulpa de copoazú puede reducir el aporte nutricional y producir enfermedades que se transmiten por alimentos manipulados inapropiadamente (Titus et al., 2018).

Se desconocen trabajos enfocados en la retención de vitamina $\mathrm{C}$ durante el procesamiento de enlatado de la pulpa de copoazú o del género Theobroma, a pesar de que la literatura muestra publicaciones disímiles en diferentes periodos. De ellas, al 2021, según la base de datos de Web of Science, se publicaron trabajos de Das Neves Selis et al., 2021; De Oliveira et al., 2021; Silva et al., 2021; Tenea \& Ortega, 2021. No obstante, existen antecedentes de que se ha evaluado la retención de vitamina C en frutas como naranja (Maeda \& Mussa, 1986), mango (Ahmed et al., 2005), manzana anacardo (Emelike \& Ebere, 2015), papaya (Avila-Gaxiola et al., 2016) 
y albaricoque (Adkison et al., 2018). A partir de este panorama, se planteó la siguiente hipótesis: el procesamiento adecuado de la pulpa de copoazú enlatado garantiza el uso comercial en empresas agroindustriales, además de permitir la reducción óptima de la vitamina C.

Es pertinente investigar la conservación de la vitamina $C$ durante el procesamiento de la pulpa de copoazú enlatada para conocer pérdida o retención total de la vitamina y, a partir de ello, determinar un protocolo adecuado en los procesos industriales para obtener pulpa pasteurizada, con el propósito de adecuar un proceso cinético que conserve el valor nutritivo y calidad alimenticia para el consumo humano. En este sentido, el objetivo del estudio fue evaluar la estabilidad de la conservación de la vitamina C en el procesamiento de la pulpa de copoazú enlatada.

\section{Metodología}

\section{Lugar de estudio}

La investigación se efectuó, durante el año 2017, en las instalaciones de la Planta Piloto de Tecnología de Alimentos y Productos Agropecuarios y en los laboratorios de físicoquímica de Calidad Total La Molina, de la Facultad de Industrias Alimentarias de la Universidad Nacional Agraria La Molina. La materia prima (copoazú) en estado fresco se obtuvo de la provincia de Tambopata, distrito Iberia, departamento de Madre de Dios (Perú).

\section{Diseño experimental y tratamientos}

Se realizó un estudio de tipo básico porque el propósito fue ampliar los conocimientos, sin fines aplicativos, de la conservación de la vitamina $C$ en el procesamiento de la pulpa de copoazú enlatada. Asimismo, se usó un nivel descriptivo, debido a que se describió sistemáticamente los experimentos hechos tanto del flujo del procesamiento tecnológico para obtener la pulpa de copoazú enlata como del tratamiento térmico. Así, el diseño que se empleó fue experimental y se constituyó en cuatro etapas.

En la etapa 1 se caracterizaron los frutos del copoazú según análisis físico-químico y análisis microbiológico, este último de acuerdo con la norma del Ministerio de Salud NTS N 071-MINSA/DIGESA-V0.1 (2008) para semiconservas de pH < 4.6.

En la etapa 2 se determinó el tratamiento térmico en la elaboración de la pulpa de copoazú enlatada. Para ello, se utilizó un envase de acero de hojalata de capacidad nominal de 15 oz/430 ml, diámetro $73 \mathrm{~mm}$ y altura $11 \mathrm{~cm}$. La tapa del envase fue de hojalata barnizada. Se utilizó el programa Data Trace Temp System para el monitoreo de la cinética de calentamiento a $100{ }^{\circ} \mathrm{C}$ (agua en ebullición) y enfriamiento a un punto equidistante de $1 / 2$ y $1 / 3$ de distancia desde la base.

Además, se determinó las unidades de pasteurización (UP) para reducir la población microbiana inicial (bacteria ácido láctica, mohos y levaduras) en el producto antes de ser sometido a tratamiento térmico. Esta reducción fue determinada según nivel de aceptabilidad de riesgo asumible propuesto por Hurtado (1987), que utiliza como referencia el Byssochlamys fulva (moho de mayor termo resistencia), cuya temperatura es $93.3^{\circ} \mathrm{C}, \mathrm{D}=1$ min y $\mathrm{Z}=8.9^{\circ} \mathrm{C}$.

Seguidamente, se sometió la pulpa a tratamiento térmico de $100{ }^{\circ} \mathrm{C}$ para determinar el tiempo de proceso. Se colocaron sensores en la pulpa de copoazú envasada para controlar el punto más frío, la temperatura del producto y el tiempo en el que se acumula la letalidad deseada. Según estos resultados, se trató térmicamente el producto (muestra por duplicado) proce- 
sando los datos a través del método general por la técnica de rectángulos y los métodos de la fórmula de Stumbo (1973), y así se pudo verificar el cumplimiento de los parámetros obtenidos en el diseño.

Por último, se evaluaron los datos generados en el tratamiento térmico mediante el método general o gráfica. Estos resultados se corroboraron con los datos generados por el programa Data Trace Temp System. Se calculó los factores de las curvas de calentamiento y enfriamiento mediante el método de Stumbo.

En la etapa 3 se determinó la variación de vitamina C durante el proceso de elaboración de la pulpa de copoazú, que pasa por etapas de mínimos procesos como el pulpeado y pasteurizado, calculando los porcentajes parciales y acumulados de cada etapa. Además, se determinó la retención de dicho compuesto en comparación con la composición inicial del fruto fresco. En la figura 1 se muestran las etapas del proceso tecnológico que se estableció para obtener la pulpa de copoazú enlatada.

Figura 1. Etapas del proceso tecnológico para la obtención de pulpa de copoazú enlatada

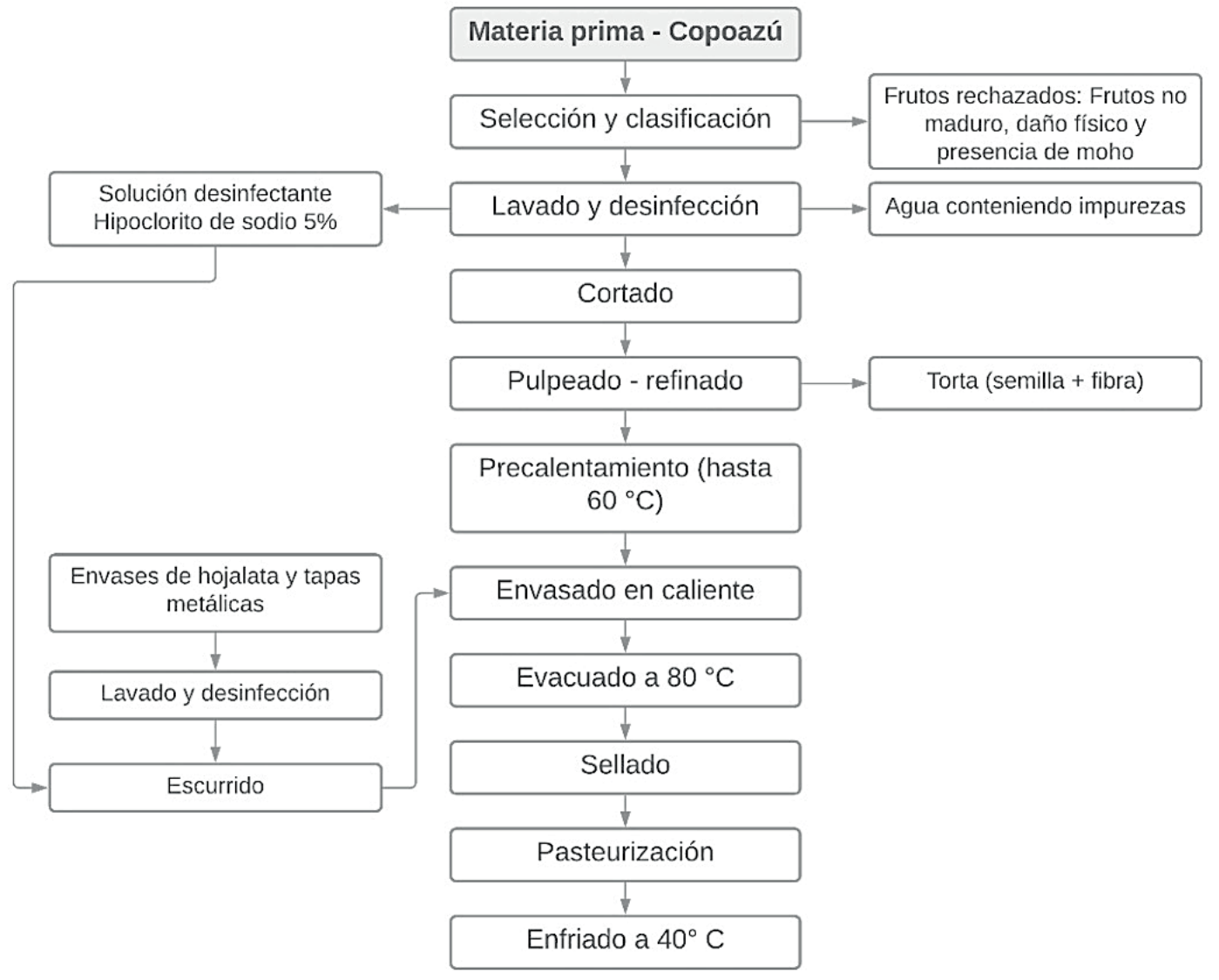

Se separaron los frutos de copoazú deteriorados por daños mecánicos, físicos o biológicos; luego fueron lavados para separar los contaminantes adheridos a la materia prima con una escobilla doméstica. Los frutos se lavaron mediante inmersión en agua con hipoclorito de sodio 0.5 \%-1.0 \%, por el lapso de 10 min, y se rompieron empleando un mazo para extraer la masa pulpa y la semilla. La etapa de pulpeado-refinado se hizo en una pulpeadora (malla con orificios 
de $2.9 \mathrm{~mm}$ de diámetro). La pulpa obtenida se refinó en una malla con orificios de $0.5 \mathrm{~mm}$ de diámetro, lo cual separó la fibra y la semilla.

En la etapa de precalentamiento se envasó la pulpa caliente en envases de hojalata. Estas fueron previamente lavadas, desinfectadas y escurridas con solución de hipoclorito de sodio $0.5 \%$. Para formar el vacío en los envases, se hizo el proceso de evacuado a través de una cadena transportadora hasta el exhauster, donde atravesó el túnel en un lapso de 5 min a temperatura de $80^{\circ} \mathrm{C}$. Finalizado el proceso, los envases fueron cerrados herméticamente.

La pulpa enlatada se sometió a la pasteurización $\left(100{ }^{\circ} \mathrm{C}\right)$ durante 40 min. Los envases se colocaron en el interior de una canastilla de metal y, posteriormente, se los introdujo en la autoclave horizontal. El producto enlatado se enfrió hasta llegar a $40^{\circ} \mathrm{C}$ aproximadamente y fue almacenado a temperatura ambiente.

Por último, la etapa 4 consistió en caracterizar la pulpa de copoazú después de su procesamiento, según análisis físicoquímico y análisis microbiológico.

\section{Análisis estadístico}

Se evaluó el efecto del procesamiento de la pulpa de copoazú enlatada en la variación de la vitamina C por triplicado. Los resultados fueron expresados como la media de tres dimensiones \pm error estándar, estos se compararon entre sí y con respecto a la materia prima. Para ello, se aplicó un diseño completamente al azar (DCA) en la etapa 2 y 3, se analizaron las diferencias estadísticas entre las medias, considerando 95 \% $(p<0.05)$ de intervalo de confianza. Se empleó un factor categórico (proceso de producción de la pulpa enlatada en copoazú) con tres niveles referentes a las etapas del proceso: materia prima, pulpeado y pasteurizado. Para el análisis de varianzas y pruebas de comparación de Tukey, se lo hizo a través del programa Statgraphics Centurion XV. II.

\section{Resultados y discusión}

\section{Análisis de materia prima}

El análisis fisicoquímico de la pulpa de copoazú fresco arrojó valores de humedad (g) equivalentes a $88.8 \pm 0.03$, proteína $0.85 \pm 0.02$, extracto etéreo (g) $0.02 \pm 0.02$, fibra (g) $1.22 \pm 0.01$, ceniza (g) $0.67 \pm 0.01$, carbohidratos (g) $13.67 \pm 0.03, \mathrm{pH} 3.00 \pm 0.02$, Brix $11.3 \pm 0.01$ y acidez (g) $1.51 \pm 0.02$. Estos resultados se asemejan a lo expuesto por Vriesmann y De Oliveira Petkowicz (2009) y Canuto et al. (2010), quienes reportaron que la pulpa presenta pH de $3.5 \pm 0.02$ y acidez entre $1.50 \%-200 \%$.

Respecto al análisis microbiológico, se obtuvo 1000 UFC/g de hongos totales y 11 × 100 000 UFC/g de levadura. El valor encontrado fue bajo, porque el fruto de copoazú tiene un caparazón que lo recubre de forma natural de daños microbiológicos y físicos (Ana L. F. Pereira et al., 2018). Este análisis se realizó para identificar la carga microbiana de la materia prima y calcular tanto los parámetros cinéticos para frutas en conservas (Byssochlamys fulva) como los datos de penetración de calor en el enlatado para determinar el valor de pasteurización requerido en el procesamiento de la conserva de copoazú. 


\section{Tratamiento térmico}

El sensor colocado a 1/3 de distancia respecto a la tapa del envase de la pulpa de copoazú arrojó un valor $P^{8,9} 100$ (pasteurización equivalente) de 4.1. En cuanto al sensor colado a 1/2, este arrojó un valor de 4.5. La tabla 1 muestra que el punto más lento de calentamiento está ubicado a 1/3 de altura desde la base (punto medio entre la base y el centro), ya que la velocidad de calentamiento (fh) es menor en dicho punto (mayor dependiente). La tabla 2 muestra que la velocidad de enfriamiento (fc) también fue menor a distancia de 1/3.

Tabla 1. Puntos más fríos durante el calentamiento

\begin{tabular}{|c|c|c|c|}
\hline \multicolumn{5}{|c|}{ Calentamiento } \\
\hline Selección del envase evaluadas & $\mathbf{1 / 3}$ desde la base & $\mathbf{1 / 2}$ altura desde la base \\
\hline $\operatorname{Tr}\left({ }^{\circ} \mathrm{C}\right)$ : Retorta & 100 & M4T11718 & M4T11741 \\
\hline Pendiente & $1 /$ fh & -0.0205 & -0.0217 \\
\hline Intercepto & $\log ($ Tr-Tpih) en t=0 & 1.8389 & 1.8516 \\
\hline \multicolumn{2}{|c|}{ To: Temperatura inicial } & 56.10 & 55.6 \\
\hline \multicolumn{2}{|c|}{ Tpih ( $\left.{ }^{\circ} \mathrm{C}\right):$ Temperatura pseudoinicial } & 47.52 & 48.71 \\
\hline \multicolumn{2}{|c|}{ fh: Velocidad del calentamiento } & 48.78 & 46.08 \\
\hline \multicolumn{2}{|c|}{ Jh: Factor de retraso } & 1.20 & 1.16 \\
\hline
\end{tabular}

Nota. Prueba realizada por duplicado

Tabla 2. Puntos más fríos durante el enfriamiento

\begin{tabular}{|c|c|c|c|}
\hline \multicolumn{4}{|c|}{ Calentamiento } \\
\hline \multicolumn{2}{|c|}{ Selección del envase evaluadas } & $\mathbf{1 / 3}$ desde la base & $\mathbf{1 / 2}$ altura desde la base \\
\hline $\operatorname{Tr}\left({ }^{\circ} \mathrm{C}\right)$ : agua de enfriamiento & 20 & M4T11718 & M4T11741 \\
\hline Pendiente & $1 /$ fh & -0.0152 & -0.0169 \\
\hline Intercepto & $\log ($ Tr-Tpih) en t=0 & 1.9768 & 1.9582 \\
\hline Tpih $\left({ }^{\circ} \mathrm{C}\right):$ Temperatura pseudoinicial & 114.80 & 110.82 \\
\hline \multicolumn{2}{|c|}{ fC: Velocidad del calentamiento } & 65.36 & 59.17 \\
\hline \multicolumn{2}{|c|}{ Jh: Factor de retraso } & 1.27 & 1.21 \\
\hline
\end{tabular}

Nota: Prueba realizada por duplicado

La figura 2 muestra las historias de tiempo-temperatura del procesamiento de pulpa de copoazú enlatada. Las nomenclaturas de las muestras fueron las siguientes: R1) M1 y M2: muestras correspondientes al primer tratamiento y R2) M3 y M4: muestra correspondientes al segundo tratamiento. 
Figura 2. Historia tiempo-temperatura de muestra de pulpa la de copoazú enlatada

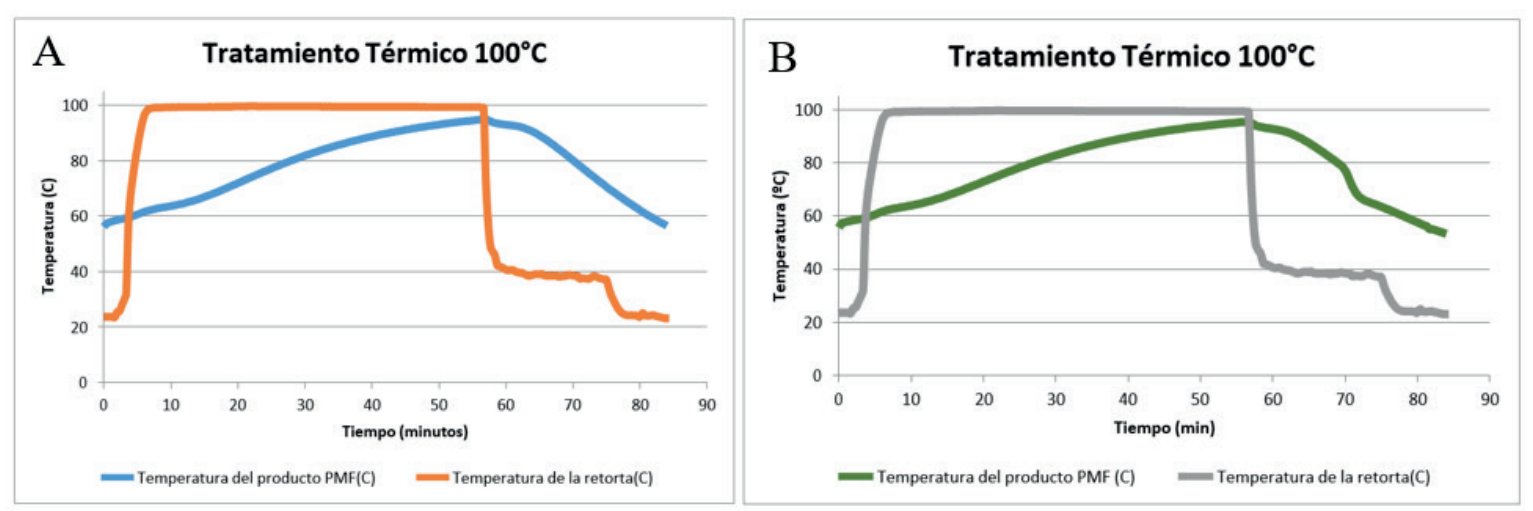

Nota: A) primera muestra. B) segunda muestra

El análisis de los gráficos permitió obtener información de los valores de Po al final de la pasteurización (tabla 3). Se observa que el tratamiento (R1) presenta un menor valor Po, correspondiente al segundo tratamiento (R2). Además, este tratamiento (R1) presenta el mayor valor fh (47.78), que permite confirmar como punto de calentamiento más lento.

Tabla 3. Características de las curvas de penetración de calor de cada tratamiento

\begin{tabular}{|c|c|c|c|c|}
\hline Tratamiento & m & fh & To & $\begin{array}{c}\text { Po final de } \\
\text { pasteurización }\end{array}$ \\
\hline R1 & -0.0205 & 48.78 & 56.10 & 4.10 \\
\hline R2 & -0.0217 & 46.08 & 55.60 & 4.50 \\
\hline
\end{tabular}

Nota: Prueba realizada por duplicado

Los parámetros de penetración de calor para el calentamiento y enfriamiento del enlatado de la pulpa de copoazú se muestran en la tabla 4. Se observa que los valores de fh y fc presentan una diferencia de 16.58 min con un $25.37 \%$ respecto al valor fh. Esta diferencia indicaría que la transferencia de calor es una combinación de convección y conducción pura. Según Sharma et al. (2003), en los productos que se calientan por convección, el punto más frío se sitúa sobre el eje vertical ligeramente más abajo del centro geométrico. Por esta razón, se confirma que el calentamiento de la pulpa de copoazú se realizó por convección lenta. Se calculó el valor Po objetivo en función del moho Byssochlamys fulva mediante la siguiente fórmula:

$$
\begin{aligned}
& U P=D_{r}(\log a-\log b) \\
& U P=P 93.3=D 93.3(\log a-\log b) \\
& P 93.3=1 \times\left(\log 1000-\log 1 \times 10^{-2}\right) \\
& P 93.3=5 \text { min }
\end{aligned}
$$


Tabla 4. Parámetros de las curvas de calentamiento y enfriamiento

\begin{tabular}{|c|c|c|c|}
\hline \multicolumn{2}{|c|}{ Calentamiento } & \multicolumn{2}{c|}{ Enfriamiento } \\
\hline fh & $48.78 \pm 1.54$ & fC & $65.36 \pm 1.78$ \\
\hline $\mathrm{To}\left({ }^{\circ} \mathrm{C}\right)$ & $56.10 \pm 0.44$ & $\mathrm{TiC}=\mathrm{Tg}\left({ }^{\circ} \mathrm{C}\right)$ & $94.90 \pm 0.45$ \\
\hline $\mathrm{Ih}=\mathrm{TR}-\mathrm{To}\left({ }^{\circ} \mathrm{C}\right)$ & $43.90 \pm 0.23$ & $\mathrm{IC}=\mathrm{Tg}-\mathrm{TC}\left({ }^{\circ} \mathrm{C}\right)$ & $74.90 \pm 0.28$ \\
\hline $\mathrm{Jhlh}=\mathrm{TR}-\mathrm{Tpsih}\left({ }^{\circ} \mathrm{C}\right)$ & $52.48 \pm 0.67$ & $\mathrm{Jclc}=\mathrm{TpiC}-\mathrm{TC}\left({ }^{\circ} \mathrm{C}\right)$ & $94.80 \pm 0.63$ \\
\hline $\mathrm{Tpsih}\left({ }^{\circ} \mathrm{C}\right)$ & $47.52 \pm 5.99$ & $\mathrm{Tpsi} \mathrm{C}$ & $114.80 \pm 5.66$ \\
\hline $\mathrm{Jh}$ & $1.20 \pm 0.32$ & $\mathrm{JC}$ & $1.27 \pm 0.38$ \\
\hline
\end{tabular}

Nota: Prueba realizada por duplicado

La UP por el método general mejorado se determinó a temperatura de $100{ }^{\circ} \mathrm{C}$, para lo cual se calculó 2.07 min, pero, teniendo en cuenta un margen de seguridad, se obtuvo 2.10 min a temperatura máxima de calentamiento del producto (figura 3).

Figura 3. Determinación de la UP por el método general

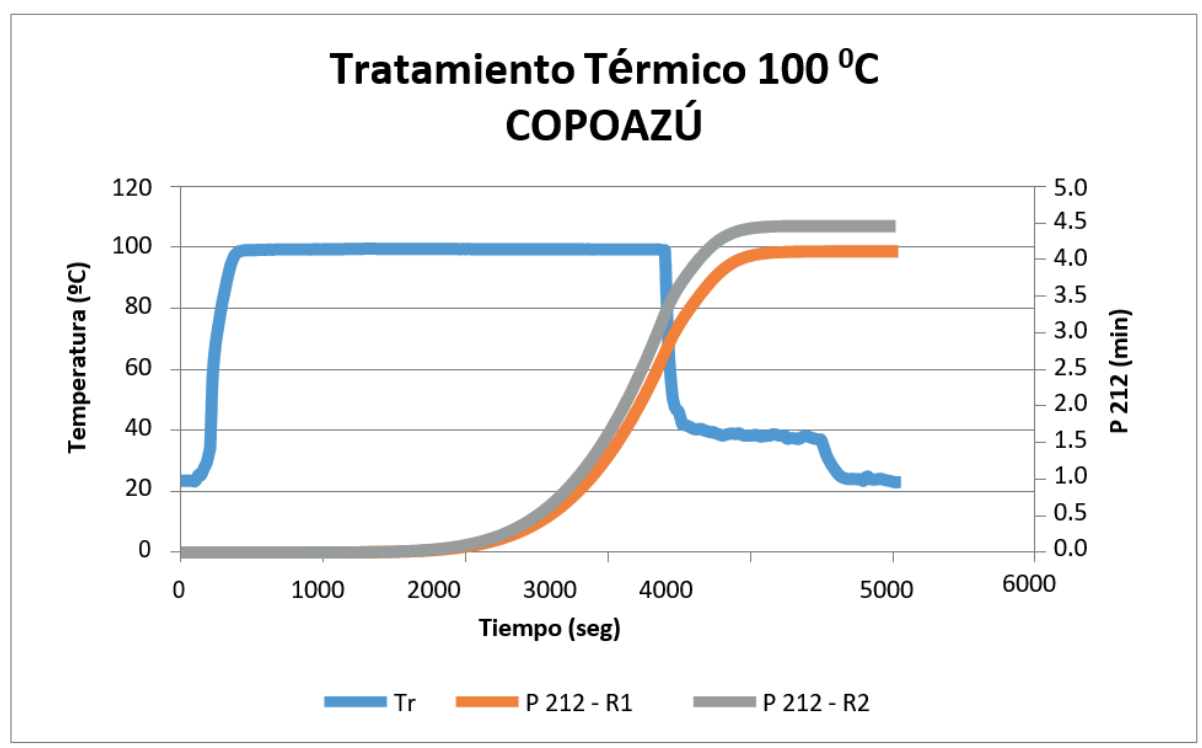

La UP, a partir del método de Stumbo para el proceso de tratamiento R1, arrojó como valor $4.02 \pm 0.34$ min. El mismo método se aplicó para determinar el tiempo de procesamiento para temperatura a $100{ }^{\circ} \mathrm{C}$, que resultó $28.35 \pm 0.34 \mathrm{~min}$. En cambio, por el método general, la suma de la letalidad conseguida en el tiempo de proceso fue de 46.60 min con los que se alcanzó 4.10 min de UP. La figura 4 muestra la acumulación de la velocidad letal (área bajo la curva) en cada intervalo de tiempo evaluado (20 segundos). 
Figura 4. Curva de la velocidad letal (L) a $100{ }^{\circ} \mathrm{C}$

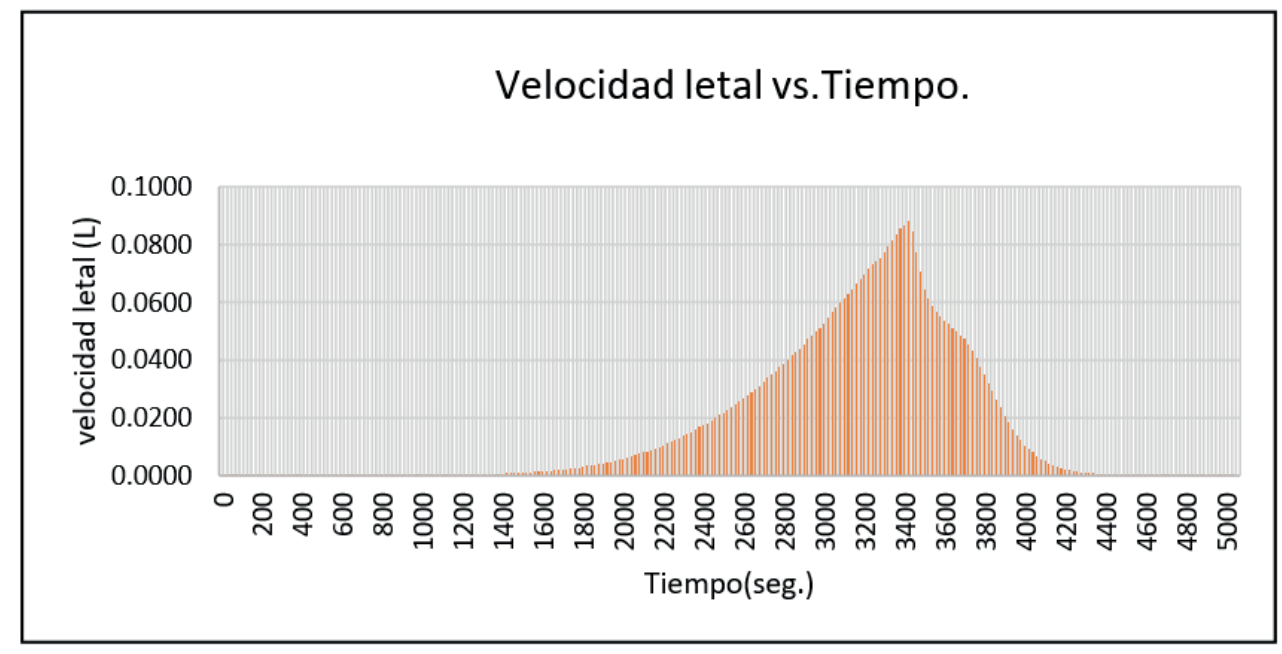

Los resultados obtenidos de la UP por ambos métodos (tabla 5) fueron corroborados con los cálculos establecidos del valor UP objetivo (5 min). Se puede ver que en el tratamiento R1 el valor obtenido por el método general fue el más cercano (4.10 min).

Tabla 5. Características de las curvas de penetración de calor de cada tratamiento

\begin{tabular}{|c|c|c|c|c|}
\hline \multirow{2}{*}{ Tratamiento } & \multicolumn{2}{|c|}{ UP (min) } & \multicolumn{2}{c|}{ Tiempo de proceso (min) } \\
\cline { 2 - 5 } & General & Stumbo & General & Stumbo \\
\hline R1 & 4.10 & 4.02 & 46.66 & 28.35 \\
\hline
\end{tabular}

En su estudio, Vieira et al. (2001) sostienen que la reducción de cargas microbianas a un nivel seguro por pasteurización evita cambios en los atributos de calidad de un producto. Así, en su investigación, Vieira et al. (2000) determinaron que los parámetros cinéticos de degradación térmica del ácido ascórbico y dehidroascórbico (25\% de pulpa y $15 \%$ de azúcar) se hallaron en la pasteurización en el rango de $60^{\circ} \mathrm{C}-100^{\circ} \mathrm{C}$ usando el método isotérmico. En relación con presente resultado, la UP se determinó a $100{ }^{\circ} \mathrm{C}$ mediante el método general y método Stumbo. Se estima que las diferencias de los procesamientos (néctar y enlatado) son criterios determinantes para el tratamiento térmico de la fruta de copoazú y estas presentan diferentes condiciones que minimizan la degradación de la vitamina $\mathrm{C}$.

La temperatura de $100{ }^{\circ} \mathrm{C}$ se justifica porque la pulpa de copoazú es un producto ácido que requiere tratamientos de pasteurización y no esterilización, dado que resultaría excesivo desde el punto de vista tecnológico y presumiblemente económico. Pokorny (2005) indica que aplicar temperatura moderada ( $100{ }^{\circ} \mathrm{C}$ como máximo) reduce los cambios negativos que se producen en la calidad nutritiva del alimento.

\section{Estabilidad de vitamina C}

La tabla 6 muestra la variación del contenido y retención de vitamina C durante el proceso de producción de la pulpa de copoazú enlatada. En el caso del fruto fresco se obtuvo $29.78 \mathrm{mg} / 100 \mathrm{~g}$ base húmeda (b.h). En la etapa de pulpeado se obtuvo una pérdida de $31.87 \%$. Esto se atribuye a la reducción de tamaño de partícula y el consecuente incremento significativo de la superficie 
expuesta al oxígeno del aire y al oxígeno contenido en las células del fruto, así como a la liberación de enzimas de los tejidos. En cuanto al contenido de vitamina $C$ durante el pulpeado, se obtuvo un valor de $20.29 \mathrm{mg} / 100 \mathrm{~g}$. Después del proceso de pasteurización, el ácido ascórbico se redujo 14.62 \% b.h. Estas reducciones en el proceso de obtención de pulpa envasada y pasteurizada suceden probablemente porque la vitamina $\mathrm{C}$ es termolábil e hidrosoluble.

La retención total fue $49.10 \% \pm 0.83$ b.h, por lo tanto, la pulpa de copoazú tratada obtuvo un valor de $14.62 \mathrm{mg} / 100 \mathrm{~g}$. Resultados superiores de retención de ácido ascórbico se constataron en el néctar de pulpa de guayaba rosada (Psidium guajava L.) con 89.3 \% (Ordóñez-Santos \& Vázquez-Riascos, 2010), así como en el néctar de la pulpa de zarzamora (Rubus fructicosus L.) con 75.9 \% (Valencia Sullca \& Guevara Pérez, 2013).

En su estudio, Emelike \& Ebere (2015) encontraron una reducción significativa de vitamina $\mathrm{C}$ en el jugo de anacardo $\left(48 \mathrm{mg} / 100 \mathrm{ml}\right.$ ) pasteurizado a $80{ }^{\circ} \mathrm{C}$. Además, Avila-Gaxiola et al. (2016) hallaron, a través del procesamiento de temperatura de retorta constante, que la retención de la vitamina $\mathrm{C}$ en puré de papaya en conserva fue de $65.71 \%$.

Estas diferencias se deben a las formulaciones empleadas, especialmente a la proporción de la dilución de la materia prima y al ajuste del pH, así como a la severidad del tratamiento térmico. No obstante, McGinnis et al. (2020) afirman que las frutas enlatadas, congeladas o frescas retienen niveles similares de nutrientes y las tres son recomendadas para el consumo humano.

Tabla 6. Variación de vitamina C (b.h) durante el procesamiento de la pulpa de copoazú enlatada

\begin{tabular}{|c|c|c|c|c|}
\hline Etapas del proceso & $\begin{array}{c}\text { Promedio } \\
\text { (mg/100 g b.h) }\end{array}$ & $\begin{array}{c}\text { Variación } \\
(\%)\end{array}$ & $\begin{array}{c}\text { Variación acu- } \\
\text { mulada (\%) }\end{array}$ & $\begin{array}{c}\text { Retención } \\
\text { (\%) }\end{array}$ \\
\hline Materia prima (Copoazú fresco) & $29.78 \pm 0.74$ & - & 00.00 & $100.00 \pm 0.00$ \\
\hline Pulpeado & $20.29 \pm 0.52$ & 31.87 & 31.87 & $68.13 \pm 1.44$ \\
\hline Pasteurizado (pulpa de copoazú) & $14.62 \pm 0.42$ & 19.03 & 50.90 & $49.10 \pm 0.83$ \\
\hline Pérdida total (\%) & $50.90 \pm 0.88$ & - & - & - \\
\hline
\end{tabular}

Nota: Análisis realizado por triplicado en base húmeda ^ desviación estándar

La tabla 7 muestra diferentes estadísticos de ácido ascórbico para cada uno de los 3 niveles de procesos de producción, teniendo en cuenta que la máxima retención es a nivel de la materia prima. La intensión principal del análisis de varianza de un factor es la de comparar las medias de los diferentes niveles.

Tabla 7. Resumen estadístico para ácido ascórbico de acuerdo con la retención por cada proceso

\begin{tabular}{|c|c|c|c|c|c|c|c|}
\hline $\begin{array}{c}\text { Tratamiento } \\
\text { o proceso de } \\
\text { producción }\end{array}$ & Recuento & Promedio & $\begin{array}{c}\text { Desviación } \\
\text { estándar }\end{array}$ & $\begin{array}{c}\text { Coeficiente de } \\
\text { variación (\%) }\end{array}$ & Mínimo & Máximo & Rango \\
\hline 1) materia prima & 3 & 100.00 & 0.0 & $0.0 \%$ & 100.0 & 100.0 & 0.0 \\
\hline 2) pulpeado & 3 & 68.13 & 0.7147 & $1.0490 \%$ & 67.36 & 68.77 & 1.41 \\
\hline 3) pasteurizado & 3 & 49.10 & 0.9118 & $1.8569 \%$ & 48.05 & 49.66 & 1.61 \\
\hline Total & 9 & 72.41 & 18.319 & $25.298 \%$ & 48.05 & 100.0 & 51.94 \\
\hline
\end{tabular}


La tabla 8 de ANOVA descompone la varianza de ácido ascórbico en dos componentes: uno entre grupos y uno dentro de grupos. El F-Ratio, que en este caso es 3314.17 igual, es el cociente entre el estimado entre grupos y el estimado dentro de grupos. Asimismo, el P-Value de la prueba $-\mathrm{F}$ es menor que 0,05 , donde existe una diferencia estadísticamente significativa entre la media de ácido ascórbico entre los niveles de proceso de producción, medidos con un nivel del $95 \%$ de confianza.

Tabla 8. ANOVA para vitamina C por tratamiento

\begin{tabular}{|c|c|c|c|c|c|}
\hline Fuente & Suma de cuadrados & GL & $\begin{array}{c}\text { Cuadrado } \\
\text { de medio }\end{array}$ & F-Ratio & P-Value \\
\hline Entre grupos & 3968.1 & 2 & 1984.05 & 3314.17 & 0.0000 \\
\hline Dentro de grupos & 359193 & 6 & 0.598656 & & \\
\hline Total (Corr.) & 3971.69 & 8 & & & \\
\hline
\end{tabular}

\section{Análisis de la pulpa de copoazú enlatada}

Los análisis fisicoquímicos realizados al producto fueron procesados a partir de parámetros que maximizan la retención de ácido ascórbico. Estos fueron peso bruto (g) $485.4 \pm 1.30$, peso drenado (g) $435.4 \pm 1.6$, peso del envase (g) $50 \pm 2.20$, presión de vacío (pulg. Hg) $13 \pm 0$,50, espacio de cabeza $(\mathrm{mm}) 8 \pm 0.50, \mathrm{pH} 3.05 \pm 0.50$, acidez (\%) $1.54 \pm 0.80$ y sólidos solubles (Brix) $12.10 \pm 0.40$.

La determinación del pH y acidez del producto terminado fue pertinente, debido a que se mantiene igual al producto fresco, es decir, 3.05 y 1.54 respectivamente, lo cual muestra una mínima variación. Por el contrario, los sólidos solubles (Brix) aumentaron significativamente con respecto al fruto fresco.

En cuanto al análisis microbiológico, luego de la pasteurización no se detectó la presencia de mohos, levaduras y bacterias ácido lácticas ( $<10 \mathrm{UFC} / \mathrm{g}$ ), por lo que se cumple con los límites (10-2 UFC/g) para productos con $\mathrm{pH}<4.6$ (semiconservas) regidos por la norma sanitaria que establece los criterios microbiológicos de calidad sanitaria e inocuidad para los alimentos y bebidas de consumo humano (NTS Nº 071-MINSA/DIGESA-V.01, 2008).

\section{Conclusiones y recomendaciones}

El ANOVA del proceso de obtención de pulpa enlatada y pasteurizada, medida en la degradación del ácido ascórbico, presenta un P-Value de la prueba -F un valor de 0.000, valor que es menor que 0.05 , por lo tanto, se concluye que existe una diferencia estadísticamente significativa entre la media de ácido ascórbico y los niveles de proceso de producción (materia prima, pulpeado y pasteurizado), medidos con un nivel del $95 \%$ de confianza. Esta diferencia existe porque la vitamina $\mathrm{C}$ es termolábil e hidrosoluble $y$, en las diferentes etapas del proceso de producción, la pulpa de copoazú es sometida a distintas reacciones por presencia de oxígeno y cambios de temperatura que afectan al contenido de vitamina $\mathrm{C}$.

Según los resultados, el ácido ascórbico (b.h) del copoazú fresco (29.78 \pm 0.74 mg/100 g) disminuyó progresivamente durante el pulpeado $(20.29 \pm 0.52 \mathrm{mg} / 100)$ y la pasteurización 
$14.62 \pm 0.42 \mathrm{mg} / 100 \mathrm{~g}$, por lo que la pérdida total es de $50.90 \pm 0.88 \mathrm{mg} / 100 \mathrm{~g}$, con una retención total de $49.10 \% \pm 0.83$.

Este estudio es el primer acercamiento a la evaluación de estabilidad de vitamina $\mathrm{C}$ en el procesamiento de la pulpa de copoazú enlatada, por lo tanto, será pertinente para la industria alimentaria. Se recomienda llevar a cabo trabajos científicos que determinen el tratamiento térmico de pasteurización sobre la retención de compuestos bioactivos y fenológicos en la pulpa enlatada de copoazú, así como su efecto en la capacidad antioxidante.

\section{Referencias}

Adkison, E. C., Biasi, W. B., Bikoba, V., Holstege, D. M., \& Mitcham, E. J. (2018). Effect of canning and freezing on the nutritional content of apricots. Journal of Food Science, 83(6), 1757-1761. https://doi. org/10.1111/1750-3841.14157

Ahmed, J., Ramaswamy, H. S., \& Hiremath, N. (2005). The effect of high pressure treatment on rheological characteristics and colour of mango pulp. International Journal of Food Science and Technology, 40(8), 885-895. https://doi.org/10.1111/j.1365-2621.2005.01026.x

Amit, S. K., Uddin, M. M., Rahman, R., Islam, S. M. R., \& Khan, M. S. (2017). A review on mechanisms and commercial aspects of food preservation and processing. Agriculture \& Food Security, 6(51). https://doi.org/10.1186/s40066-017-0130-8

Avila-Gaxiola, E., Delgado-Vargas, F., Zazueta-Niebla, J., López-Angulo, G., Vega-García, M., \& Caro-Corrales, J. (2016). Variable retort temperature profiles for canned papaya puree. Journal of Food Process Engineering, 39(1), 11-18. https://doi.org/10.1111/jfpe.12194

Badui Dergal, S. (2006). Química de los alimentos (4. ${ }^{\text {a } e d .) . ~ P e a r s o n ~ E d u c a c i o ́ n . ~}$

Bouzari, A., Holstege, D., \& Barrett, D. M. (2015). Vitamin retention in eight fruits and vegetables: A comparison of refrigerated and frozen storage. Journal of Agricultural and Food Chemistry, 63(3), 957-962. https://doi.org/10.1021/jf5058793

Canuto, G. A. B., Xavier, A. A. O., Neves, L. C., \& Benassi, M. de T. (2010). Caracterização físico-química de polpas de frutos da Amazônia e sua correlação com a atividade anti-radical livre. Revista Brasileira de Fruticultura, 32(4), 1196-1205. https://doi.org/10.1590/S0100-29452010005000122

Cole, M. B., Augustin, M. A., Robertson, M. J., \& Manners, J. M. (2018). The science of food security. npj Science of Food, 2(14). https://doi.org/10.1038/s41538-018-0021-9

Costa, M. P., Frasao, B. S., Silva, A. C. O., Freitas, M. Q., Franco, R. M., \& Conte-Junior, C. A. (2015). Cupuassu (Theobroma grandiflorum) pulp, probiotic, and prebiotic: Influence on color, apparent viscosity, and texture of goat milk yogurts. Journal of Dairy Science, 98(9), 5995-6003. https://doi. org/10.3168/jds.2015-9738

Das Neves Selis, N., De Oliveira, H. B. M., Dos Anjos, Y. B., Leão, H. F., Sampaio, B. A., Correia, T. M. L., Reis, M. M., Brito, T. L. S., Almeida, C. F., Pena, L. S. C., Brito, L. F., Ornelas, R. M., Santos, T. T., Campos, G. B., Timenetsky, J., Cruz, M. P., Da Costa, A. M., Yatsuda, R., Uetanabaro, A. P. T., \& Marques, L. M. (2021). Gardnerella vaginalis and Neisseria gonorrhoeae are effectively inhibited by lactobacilli with probiotic properties isolated from Brazilian cupuaçu (Theobroma grandiflorum) Fruit. BioMed Research International, 2021, 1-15, artículo 6626249. https://doi.org/10.1155/2021/6626249

De Oliveira, P. D., Da Silva, D. A., Pires, W. P., Bezerra, C. V., Da Silva, L. H. M., \& Da Cruz Rodrigues, A. M. (2021). Enzymatic interesterification effect on the physicochemical and technological properties of cupuassu seed fat and inaja pulp oil blends. Food Research International, 145, artículo 110384. https://doi.org/10.1016/j.foodres.2021.110384

Emelike, N. J. T., \& Ebere, O. C. (2015). Effect of packaging materials, storage conditions on the vitamin C and $\mathrm{pH}$ value of cashew apple (Anacardium occidentale L.) juice. Journal of Food and Nutrition Sciences, 3(4), 160-165. https://doi.org/10.11648/j.jfns.20150304.14 
Ghoshal, G. (2018). Emerging Food Processing Technologies. En Food Processing for Increased Quality and Consumption (pp. 29-65). Elsevier. https://doi.org/10.1016/B978-0-12-811447-6.00002-3

Gondim, T. M. de S., Thomazini, M. J., Cavalcante, M. de J. B., \& Souza, J. M. L. (2001). Aspectos da produção de cupuaçu. Documento 67. Embrapa Acre. https://www.infoteca.cnptia.embrapa.br/ bitstream/doc/498481/1/doc67.pdf

Hurtado, P. (1987). Procesos tecnológicos de conservación de frutas y hortalizas y su almacenamiento. Cartagena, Colombia.

Maciel, R. M. G., Lima, S. B., Costa, J. M. C., \& Afonso, M. R. A. (2020). Influência da maltodextrina nas propriedades de escoamento do pó da polpa de cupuaçu. Brazilian Journal of Development, 6(2), 5829-5839. https://doi.org/10.34117/bjdv6n2-039

Maeda, E. E., \& Mussa, D. M. D. N. (1986). The stability of vitamin C (L-ascorbic acid) in bottled and canned orange juice. Food Chemistry, 22(1), 51-58. https://doi.org/10.1016/0308-8146(86)90008-7

McGinnis, M. J., Gustashaw, K. A. R., \& Painter, J. E. (2020). Fruit myth or fact. Nutrition Today, 55(6), 322327. https://doi.org/10.1097/NT.0000000000000447

NTS Nº 071-MINSA/DIGESA-V.01 (2008) Norma sanitaria que establece los criterios microbiológicos de la calidad sanitaria e inocuidad para los alimentos y bebidas de consumo humano. Resolución Ministerial No 591-2008/MINSA, 27 de agosto. https://bit.ly/3ub9mig

Ordóñez-Santos, L. E., \& Vázquez-Riascos, A. (2010). Effect of processing and storage time on the vitamin $\mathrm{C}$ and lycopene contents of nectar of pink guava (Psidium guajava L.). Archivos Latinoamericanos de Nutrición, 60(3). https://www.alanrevista.org/ediciones/2010/3/art-10/

Peña Valdeiglesia, J., \& Alegre Orihuela, J. C. (2017). Tipificación de prototipos de sistemas de producción agroforestal en la provincia de Tambopata, Madre de Dios. Aporte Santiaguino, 10(2), 233-244. https://doi.org/10.32911/as.2017.v10.n2.166

Pereira, Ana L. F., Abreu, V. K. G., \& Rodrigues, S. (2018). Cupuassu-Theobroma grandiflorum. Exotic Fruits, 159-162. https://doi.org/10.1016/b978-0-12-803138-4.00021-6

Pereira, Ana Lúcia Fernandes, Feitosa, W. S. C., Abreu, V. K. G., Lemos, T. de O., Gomes, W. F., Narain, N., $\&$ Rodrigues, S. (2017). Impact of fermentation conditions on the quality and sensory properties of a probiotic cupuassu (Theobroma grandiflorum) beverage. Food Research International, 100(part 1), 603-611. https://doi.org/10.1016/j.foodres.2017.07.055

Pokorny, J. (2005). Antioxidantes de los alimentos: Aplicaciones prácticas. Acribia.

Pugliese, A. G., Tomas-Barberan, F. A., Truchado, P., \& Genovese, M. I. (2013). Flavonoids, Proanthocyanidins, vitamin C, and antioxidant activity of Theobroma grandiflorum (cupuassu) pulp and seeds. Journal of Agricultural and Food Chemistry, 61(11), 2720-2728. https://doi.org/10.1021/jf304349u

Ramos, S., Salazar, M., Nascimento, L., Carazzolle, M., Pereira, G., Delforno, T., Nascimento, M., De Aleluia, T., Celeghini, R., \& Efraim, P. (2020). Influence of pulp on the microbial diversity during cupuassu fermentation. International Journal of Food Microbiology, 318, artículo 108465. https://doi. org/10.1016/j.ijfoodmicro.2019.108465

Salgado, J. M., Rodrigues, B. S., Donado-Pestana, C. M., Dos Santos Dias, C. T., \& Morzelle, M. C. (2011). Cupuassu (Theobroma grandiflorum) peel as potential source of dietary fiber and phytochemicals in whole-bread preparations. Plant Foods for Human Nutrition, 66(4), 384-390. https:// doi.org/10.1007/s11130-011-0254-0

Sharma, S. K., Mulvaney, S. J., \& Rizvi, S. S. H. (2003). Ingeniería de alimentos: Operaciones unitarias y prácticas de laboratorio. Limusa.

Silva, A. G. M., Lima, S. C. G., De Oliveira, P. D., Moraes, M. Dos S., Guimarães, C. M. C., Silva, J. A. R., Garcia, A. R., Nahúm, B. de S., Neres, L. de S., Noronha, G. N., \& Lourenço Júnior, J. de B. (2021). Production, chemical composition, and fatty acid profile of milk from buffaloes fed with cupuaçu (Theobroma grandiflorum) cake and murumuru (Astrocaryum murumuru) cake in the Eastern Amazon. Animal Science Journal, 92(1), artículo e13576. https://doi.org/10.1111/asj.13576

Stumbo, R. (1973). Thermobacteriology in food processing (2. ${ }^{\mathrm{a}}$ ed.). Academic Press. 
Tenea, G. N., \& Ortega, C. (2021). Genome characterization of Lactiplantibacillus plantarum strain UTNGt2 originated from Theobroma grandiflorum (white cacao) of Ecuadorian Amazon: Antimicrobial peptides from safety to potential applications. Antibiotics, 10(4), 383. https://doi.org/10.3390/ antibiotics 10040383

Titus, D., James Jebaseelan Samuel, E., \& Mohana Roopan, S. (2018). Importance of food science and technology-way to future. En S. M. Roopan \& G. Madhumitha (Eds.), Bioorganic phase in natural food: An overview (pp. 11-23). Springer. https://doi.org/10.1007/978-3-319-74210-6_2

Valencia Sullca, C. E., \& Guevara Pérez, A. (2013). Variación de la capacidad antioxidante y compuestos bioactivos durante el procesamiento del néctar de zarzamora (Rubus fructicosus L.). Revista de la Sociedad Química del Perú, 79(2). http://www.scielo.org.pe/pdf/rsqp/v79n2/a04v79n2.pdf

Vieira, M. C., Teixeira, A. A., \& Silva, C. L. M. (2000). Mathematical modeling of the thermal degradation kinetics of vitamin $C$ in cupuaçu (Theobroma grandiflorum) nectar. Journal of Food Engineering, 43(1), 1-7. https://doi.org/10.1016/S0260-8774(99)00121-1

Vieira, M. C., Teixeira, A. A., \& Silva, C. L. M. (2001). Kinetic parameters estimation for ascorbic acid degradation in fruit nectar using the Partial Equivalent Isothermal Exposures (PEIE) Method under non-isothermal continuous heating conditions. Biotechnology Progress, 17(1), 175-181. https:// doi.org/10.1021/bp000132w

Vriesmann, L. C., \& De Oliveira Petkowicz, C. L. (2009). Polysaccharides from the pulp of cupuassu (Theobroma grandiflorum): Structural characterization of a pectic fraction. Carbohydrate Polymers, 77(1), 72-79. https://doi.org/10.1016/j.carbpol.2008.12.007 